\title{
"eHospital - Dompe" project - the story of the transformation of a district hospital in Sri Lanka
}

\author{
Dr. K. B. Sampath Kulathilaka MBBS, Cet. in Health Programme Management \\ Medical Officer In-Charge of "eHospital-Dompe" Project, District Hospital, Dompe, Sri Lanka \\ E-mail Address: drsampathkulathilaka@gmail.com
}

Sri Lanka Journal of Bio-Medical Informatics 2013;4(2):40-43

doi: http://dx.doi.org/10.4038/sljbmi.v4i2.5757

\begin{abstract}
The "eHospital - Dompe" project was aimed making a District Hospital in Sri Lanka more efficient through adaptation of ICT solutions and appropriate change management. In this case report we describe that process.
\end{abstract}

Keywords - eHospital Dompe; Government Hospital; ICT; Change Management

\section{In the past}

This project was conceived in 2010. At that time the District Hospital, Dompe was an ordinary government hospital. The hospital was disorganized, overcrowded and there were unnecessary delays that compromised total patient care. The author, the hospital administration, and the hospital staff wanted to change the situation and make the hospital more efficient.

\section{New concepts}

Under the guidance of Dr. A.L.A.L Padmasiri (Regional Director of Health Services, Gampaha) and Dr. Hajitha Piyantha Kumara (Medical Officer who was the Acting Director of the Hospital), the author, with the help of all categories of hospital staff launched a programme to provide efficient health care. The programme included attitude changing, improving moral, building team spirit and obtaining optimal use of the abilities of staff. In addition re-arrangement of the hospital setting, provision of specific accesses to each service points - especially emergency access and improving the quality of care provided - were also included into the programme. A new patient registration desk, Queue Management Centre, and a Reception desk were established and 5s concepts were introduced. In addition, an uninterrupted power supply solution was installed with the assistance of donors in the community.

\section{The ICT Solution - Background}

We believed that an information technology solution would help make the hospital more efficient and approached the Information and Communication Technology Agency of Sri Lanka (ICTA) for support. The ICTA supported us with a grant of Rs.4,150,000.00 for wired networking, computer hardware, and software. This was facilitated by Mr. Wasantha Deshapriya (Director, re-engineering government), Ms. Chitrangani Mubharak (Head, eSociety initiative) and Mr. Shriyananda Rathnayaka (Project Manager) of the ICTA.

We visited Base Hospital Karawanella where an ICT system was already in place. This enabled us to get an idea of the software that was available. We found that the software had 
to be customized to make it suitable for our hospital. After extensive discussions Lunar Technologies, Kalutara, under the direct guidance of the author and his team, developed a new system [Hospital Health Information Management System (HHIMS) Version 1.2]. This system has undergone several revisions since then. It is now available as free and open source software that could be downloaded, installed, and used by anyone. This was facilitated by the ICTA.

To deploy the software solution, we networked all the Units of the Hospital, and bought an in-house server [Processor - Quad core E5530 Xeon 2.4 Ghz]. All the networking was wired and was done by Sri Lanka Telecom Services. All the service points/units were provided with a computer [A total of 41 computers: 31 were desktops (processor - dual core) and 10 were laptops (processor i3)]. Sri Lanka Telecom Services was also the hardware provider.

The system was inaugurated on 27 December 2011 under the patronage of Mr. Chandana Rodrigo (Secretary, Ministry of Health, Western Province) and Dr. Amal Harsha De Silva (Provincial Director of Health Services, Western Province), and Dr. A.L.A.L Padmasiri (Regional Director of Health Services, Gampaha).

\section{The IT Solution - Process}

The system enables tracking of the patient throughout a hospital visit. When a patient comes to the outpatient department (OPD), he/she is registered in the system. The system generates a "Patient Identification Number" and a "Patient specific Bar-Code". The patient is issued a "Bar-Coded Patient's Health Card". The patient takes the card and goes to the "Electronic Queue Management Centre". At the Center he/she is given the "Today's Token" assigning $\mathrm{him} / \mathrm{her}$ to the correct place in the queue and correct doctor's chamber. This way the correct doctor will see him/her when his/her turn comes.

Every doctor uses a laptop and a bar-code scanner. The doctor swaps the "Bar-Coded Patient's Health Card" with the barcode scanner and immediately retrieves the patient demographic data, past medical and surgical histories, allergic history, etc. At the end of the consultation, the doctor enters the diagnosis, the prescription containing the drugs prescribed, and the procedures/lab tests to be performed on the patient (if any). The patient is then sent to the relevant unit to for treatment/lab tests.

As a result, when the doctor orders drugs the patient is sent to the dispensary; when the doctor orders a procedure the patient is sent to the dressing room, injection room or the emergency treatment unit as necessary; and when the doctor orders a lab test, the patient is sent to the sample collection centre. At all these places, the relevant staff can get the information about the patient by swiping the "Bar-Coded Patient's Health Card". The dispensary and drug stores, the dressing room, the injection room, and the emergency treatment unit are all connected to the system. So when what was ordered by the doctor has been done or the results are available, as in the case of laboratory tests, the doctor can immediately know it. If a patient is admitted to a ward for treatment, then his medical records are updated at the time of discharge and a system generated diagnosis card is issued.

An additional feature of the system is immediate notification of notifiable diseases via email. Stand alone clinics in the hospital, such as the Medical Clinic, Family Medical Clinic, and the Screening Clinic for Non Communicable Diseases (NCDs) are also linked to the system. The MO's office is also linked to the system and as a result, he can monitor the hospital from his 
room.

All the daily reports (OPD register, Drugs dispensed, etc.) are generated by the system at the end of the day. The system automatically generates backups at $1 \mathrm{pm}, 5 \mathrm{pm}$ and 12 mid-night and save it in the server and computer placed in the DMO's office (remote location) ensuring data security. In addition, a CD is burnt daily. Hard copies of the OPD register and drugs dispensed are kept in the medical records room and with the chief pharmacist respectively for references and auditing purposes.

The system itself has other built in security features to ensure data security as the system contains sensitive patient data. All the patient data are stored in an in-house server and not in individual computers. The server is kept in a secured place and under the direct supervision of the doctor - on call. Every user is given an individual user name and password. To prevent unauthorized access user data access levels have been pre-defined. The data is also encrypted to make it more secure.

As stated above the doctors and other health care providers can gain access to the patient data only by swiping the "Bar-Coded Patient's Health Card". This was privacy is ensured and potential loss of confidentiality is minimised.

\section{Advantages}

The system has been in operation for approximately 22 months at the time of writing this paper. The system holds data of approximately 44,000 patients. The OPD has become methodical, efficient and trouble free after the implementation of the IT solution. Two minor staff officers who manually did daily registrations have been re assigned for other tasks now, saving manpower.

The OPD staff has benefited from the electronic queue management system, and the system generated daily report. The patients are easily managed now as they have faith in the system.

The doctors have benefited by getting accurate, comprehensive past histories with medication, investigations and demographic data. They know the availability of drugs immediately. They can plan the patient's management and easily retrieve lab reports. With all those information doctors can make a more accurate diagnosis. At the same time they are directed for rational use of drugs, especially antibiotics. Doctors can also make sure that the ordered procedure was performed correctly at the relevant unit. The doctors can have a look at statistics for their personal interest (eg. the number of patients treated by him/her).

The nurses in each unit have benefited by their tasks being made easy as they get clear orders from doctors in advance of the patients' arrival, which helps them to get ready for treatment. In addition, they get system generated daily summaries. The pharmacists /dispensers are able to get legible prescriptions with automatic calculation of the number of tablets, making their duty easier. In addition, automatic stock balancing has reduced their workload. The Medical Laboratory Technicians receives properly labeled samples.

The administrator is also benefited as he/she can monitor the whole process sitting in his/her room. Obtaining statistical data is made easy by the system and it helps to utilize manpower optimally. But, the main advantage is for the patients. With the help of the system they get better care. 


\section{Challenges}

There are two main challenges in the Sri Lankan health sector that prevents embracing of IT. First is the complexity of the health sector and the other is poor knowledge and attitudes towards IT. We launched several programmes to overcome the later problem. Several lectures, workshops, competitions (eg. Best Worker Competition), 5S programmes, productivity programmes (Institutional/Home/Green Productivity) and out bound training were arranged for all health staff with the view to changing their attitudes. All of our staff has been given basic computer training under the guidance and direct involvement of the Faculty of Computer Science and Engineering, University of Moratuwa. Dr. Chandana Gamage, Head of the Department, guided the programme. Then we selected 60 staff members who were directly involved with the system and provided them with software training. A selected small group was trained on basics of hardware as well. They were refreshed and guided regularly by the author. Small user group discussions were conducted to understand and improve the usability. The patients got used to the new system rapidly. They were intelligent enough to understand the value of this project and it made our task easier.

\section{The way forward}

The system has been in successful operation for 22 months by now. We always try to improve the software and its usability to improve the quality of health care provided by our hospital. Improving the patient's health card with the Health Identification Number (HIN) proposed by the Ministry of Health is one of our priority expectations. We are working hard to establish an appointment system over the phone for our hospital to ensure a more patient friendly service. Infrastructure development within our hospital is also necessary for the sustainability of the project. The electronic Bed Head Ticket and inter connectivity with other hospitals are needed to have an effective electronic transformation and these are within our reach.

\section{Conclusion}

With the experience gathered through the "eHospital-Dompe" project, we can conclude that appropriate use of ICT can definitely contribute to improvement in the quality of care provided in the Sri Lankan healthcare sector.

\section{Electronic Resources}

1. eHospital- Dompe Project in YouTube. Available at: http://www.youtube.com/watch?v=-YqujXDfHHQ

2. Hospital Health Information Management System (HHIMS) Can be downloaded from git://gitrepo.icta.lk/hhims/hhims.git 